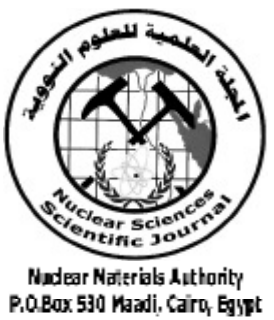

ISSN 2314-5609

Nuclear Sciences Scientific Journal

7, 205-213

2018

http://www.ssnma.com

\title{
EDGE DETECTION ENHANCED TECHNIQUE OF SATELLITE IMAGES USING DESIGN DIGITAL FILTER
}

\author{
RAAFAT M. MOHAMED and AHMED I. KAMEL \\ Nuclear Materials Authority, P. O. Box 530 El Maadi, Cairo, Egypt.
}

\begin{abstract}
Most image's interpreters are concerned with recognizing linear features in images. For geological purposes, faults, joints, features, dykes and geological contacts are the main linear features which must be interpreted. The linear features are formed by edges, where some edges are marked by pronounced differences in brightness and become ready recognized. This paper deals with satellite image edge detection techniques. Edge detection is the name for a set of mathematical methods which aim at identifying points in a satellite image at which the image brightness changes sharply or, more formally, has discontinuities. These points are typically organized into a set of curved line segments termed edges. Important features can be extracted from the edges of an image (e.g., corners, geological boundary, linesand curves). In this paper, a novel edge detection technique which computes edges of satellite image using two dimensional (2D) design digital filter is presented and applied on Gabal Gattar granitic batholith to enhance boundaries between granite and surrounded rocks in one hand, and between the granitic phases on another hand, as well as, enhance the shape of granitic masses, and faults, dykes, fractures cutting them. Here the proposed methodology is compared with other edge detectors. Filters (Sobel, Roberts, Prewitt, Canny and Log filters) as applied on G. Gattar granitic mass. This study reveals that the new proposed (Raafat filter) porttery the contacts and linear features more obvious than the other operators.
\end{abstract}

\section{INRTODUCTION}

Edge detection is a fundamental tool in image processing, image analysis and image pattern recognition, particularly in the areas of feature detection and feature extraction. Generally, due to the variation of image features such as brightness, edges can be recognized. Mainly the edges are indication of the discontinuities of image intensity function. In image processing, edges give valuable and very important information towards human image understanding. Edge detection has become very serious challenge to the scientist as well as researchers related to image processing.

Contrast enhancement many emphasize brightness difference associated with some features, but this procedure is not specific for linear features because elements of the scene are enhanced equally, not just the linear elements. Edge enhancement image attempt to presence both local contrast and low frequency brightness information. They are produces by adding back all or a portion of the gray levels in the original image to a high frequency component image of the same scene (Lillsand and Kiefer, 2000). The main objective of edge detection is to indicate the points in a digital image where the luminous intensity changes very sharply. Due to sharp changes in image properties, it reflects the valuable events and changes in several properties. For discontinuities, there could be a 
number of reasons, such as lighting conditions, type of material surface, texture, object geometry etc., and their mutual interaction. Edge detection methodology is a process of detection of this type of discontinues in an image. Generally, the implementation of derivative operator on intensity image generates another image, which is called as gradient image due to its rate of intensity variation. The major structural properties can be represented in a transparent manner of an image due to the edge detection mechanism. Many methods can be implemented for edge detection. There are many traditional operators such as Sobel operator, Prewitt operator, Laplacian of Gaussian operator, Canny operator and Robert operator which are used for edge detection of images.

\section{METHODOLGY}

Edge enhancement is implemented, firstly, produced a high frequency component image containing the edge information followed by added back all or a fractures of gray level in each pixel of the original scene to the high frequency component image. The final image containing local contrast enhancement of high frequency features (Lillsand and Kiefer, 2000). Some linear features occurred as narrow linear against a background, while linear contrast is located between adjacent areas of different brightness in images. These linear features are formed by edges. In images, some edges are easily recognized due to their pronounced differences in brightness, while other which marked by subtle brightness differences are difficult to recognize. Digital filters have been developed specifically to enhance edges in image. These filters fall into two categories, directional and non-directional filters (Sabin, 1996).

Directional filters aimed at emphasizing edges in image data. It is procedure that systematically compare each pixel in an image to one of its immediately adjacent neighbors and displays the difference in terms of gray levels of an output image. This process is mathematically akin to determining the first derivative of gray levels with respect to a given direction (horizontal, vertical and diagonal) (Lillsand and Kiefer, 2000). In order to design the 2D digital filter, we used a second order polynomial as follows:

\section{Second Order Polynomial}

Suppose that, we have $5 \times 5$ data points and wish to fit the observed data, in some sense, by the following polynomial:

$$
a_{00}+a_{10} x+a_{01} y+a_{11} x y+a_{20} x^{2}+a_{02} y^{2}=F(x, y)
$$

where $a_{00}, a_{10}, a_{01}, a_{11}, a_{20}$, and $a_{02}$ are the coefficients to be determined. The computations for the above procedure can be relatively easy, if the potential field data function $F\left(x_{i}, y_{i}\right)$ for each value of $\mathrm{x}$ and $\mathrm{y}$ where, the $\mathrm{x}$ 's and y's are equally spaced and arranged symmetrically around the calculation point i.e. $x_{i}=-2,-1,0,1,2$ and $y_{i}=-$ 2, -1, 0, 1, 2 such that $\sum_{i=-2}^{2} x_{i}=0$ and $\sum_{i=2}^{2} y_{i}=0$

The variables of equation (1) are the coefficients $a_{00}, a_{10}, a_{01}, a_{11}, a_{20}$, and $a_{02}$ of the plane. To find a minimum, we naturally differentiate with respect to $a_{00}, a_{10}, a_{01}, a_{11}, a_{20}$, and $a_{02}$ then set the resulting expressions equal to zero. This process (normalization) gives the following equations:

$$
\begin{aligned}
& a_{00} n+a_{11} \sum x+a_{10} \sum y+a_{11} \sum x y+a_{20} \sum x^{2}+a_{102} \sum y^{2}=\sum F \\
& a_{00} \sum x+a_{10} \sum x^{2}+a_{01} \sum x y+a_{11} \sum x^{2} y+a_{20} \sum x^{3}+a_{02} \sum x y^{2}=\sum F x \\
& a_{00} \sum y+a_{10} \sum x y+a_{01} \sum y^{2}+a_{11} \sum x y^{2}+a_{20} \sum x^{2} y+a_{02} \sum y^{3}=\sum F y \\
& a_{10} \sum x^{2}+a_{10} \sum x^{3}+a_{111} \sum x^{2} y+a_{11} \sum x^{3} y+a_{10} \sum x^{4}+a_{010} \sum x^{2} y^{2}=\sum F x^{2} \\
& a_{00} \sum x y+a_{10} \sum x^{2} y+a_{01} \sum x y^{2}+a_{11} \sum x^{2} y^{2}+a_{20} \sum x^{3} y+a_{02} \sum x y^{3}=\sum F x y \\
& a_{00} \sum y^{2}+a_{10} \sum x y^{2}+a_{01} \sum y^{3}+a_{11} \sum x y^{3}+a_{20} \sum x^{2} y^{2}+a_{02} \sum y^{4}=\sum F y^{2}
\end{aligned}
$$

In our case of study $\mathrm{n}=25$ then,

$$
\sum x=0, \sum y=0, \sum x^{2}=50, \sum y^{2}=50, \sum x y=0,
$$

$\sum x^{2} y=0, \quad \sum x^{3}=0, \quad \sum x y^{2}=0, \quad \sum x^{2} y=0, \quad \sum y^{3}=0, \quad \sum x^{3} y=0, \quad \sum x^{4}=170$, $\sum x^{2} y^{2}=100, \sum x y^{3}=0, \sum y^{4}=170$. Substituting in the above set of simultaneous equation, we get:

$$
\begin{aligned}
& \text { (25) } a_{00}+(0) a_{10}+(0) a_{01}+(0) a_{11}+(50) a_{20}+(50) a_{02}=\sum F \\
& \text { (0) } a_{00}+(50) a_{10}+(0) a_{01}+(0) a_{11}+(0) a_{20}+(0) a_{02}=\sum F x \\
& \text { (0) } a_{00}+(0) a_{10}+(50) a_{01}+(0) a_{11}+(0) a_{20}+(0) a_{02}=\sum F y
\end{aligned}
$$


$(50) a_{00}+(0) a_{10}+(0) a_{01}+(0) a_{11}+(170) a_{20}+(100) a_{02}=\sum F x^{2}$

$(0) a_{00}+(0) a_{10}+(0) a_{01}+(100) a_{11}+(0) a_{20}+(0) a_{02}=\sum F x y$

$(50) a_{00}+(0) a_{10}+(0) a_{01}+(0) a_{11}+(100) a_{20}+(170) a_{02}=\sum F y^{2}$

From equations (8)-(13), we get that the values of the coefficients $a_{00}, a_{10}, a_{01}, a_{11}, a_{20}$, and $a_{02}$ of the plane are:

$a_{00}=\left(\sum F-(50) a_{20}-(50) a_{02}\right) / 25$,

$a_{10}=\sum F x / 50, a_{01}=\sum F y / 50, a_{11}=\sum F x y / 100, a_{20}=\left(\sum F x^{2}-(2) \sum F\right) / 70$ and $a_{02}=\left(\sum F y^{2}-(2) \sum F\right) / 70$.

So, finally we find that the digital filter takes the following form:

$a_{00}=\left[\begin{array}{ccccc}-0.074 & 0.011 & 0.04 & 0.011 & -0.074 \\ 0.011 & 0.097 & 0.126 & 0.097 & 0.011 \\ 0.04 & 0.126 & 0.154 & 0.126 & 0.04 \\ 0.011 & 0.097 & 0.126 & 0.097 & 0.011 \\ -0.074 & 0.011 & 0.04 & 0.011 & -0.074\end{array}\right] F_{i j}$

$a_{10}=\left[\begin{array}{ccccc}-0.04 & -0.04 & -0.04 & -0.04 & -0.04 \\ -0.02 & -0.02 & -0.02 & -0.02 & -0.02 \\ 0 & 0 & 0 & 0 & 0 \\ 0.02 & 0.02 & 0.02 & 0.02 & 0.02 \\ 0.04 & 0.04 & 0.04 & 0.04 & 0.04\end{array}\right] F_{i j}$

$a_{01}=\left[\begin{array}{lllll}-0.04 & -0.02 & 0 & 0.02 & 0.04 \\ -0.04 & -0.02 & 0 & 0.02 & 0.04 \\ -0.04 & -0.02 & 0 & 0.02 & 0.04 \\ -0.04 & -0.02 & 0 & 0.02 & 0.04 \\ -0.04 & -0.02 & 0 & 0.02 & 0.04\end{array}\right] F_{i j}$

$a_{11}=\left[\begin{array}{ccccc}0.04 & 0.02 & 0 & -0.02 & -0.04 \\ 0.02 & 0.01 & 0 & -0.01 & -0.02 \\ 0 & 0 & 0 & 0 & 0 \\ -0.02 & -0.01 & 0 & 0.01 & 0.02 \\ -0.04 & -0.02 & 0 & 0.02 & 0.04\end{array}\right] F_{i j}$ $a_{20}=\left[\begin{array}{ccccc}0.029 & 0.029 & 0.029 & 0.029 & 0.029 \\ -0.014 & -0.014 & -0.014 & -0.014 & -0.014 \\ -0.029 & -0.029 & -0.029 & -0.029 & -0.029 \\ -0.014 & -0.014 & -0.014 & -0.014 & -0.014 \\ 0.029 & 0.029 & 0.029 & 0.029 & 0.029\end{array}\right] F_{i j}$

$$
a_{02}=\left[\begin{array}{lllll}
0.029 & -0.014 & -0.029 & -0.014 & 0.029 \\
0.029 & -0.014 & -0.029 & -0.014 & 0.029 \\
0.029 & -0.014 & -0.029 & -0.014 & 0.029 \\
0.029 & -0.014 & -0.029 & -0.014 & 0.029 \\
0.029 & -0.014 & -0.029 & -0.014 & 0.029
\end{array}\right] F_{i j}
$$

If we fit this data by a plane surface polynomial in least-squares sense (equation. (1)), then the first derivative in X direction can be represented by the following equation:

$$
F_{x}=a_{10}+a_{11} y+2 a_{20} x
$$

where $a_{10}$ is the gradient in $\mathrm{X}$ direction. By the same manner the firstderivative in $\mathrm{Y}$ direction could be computed as:

$$
F_{y}=a_{01}+a_{11} x+2 a_{02} y
$$

where $a_{01}$ is the gradient in $\mathrm{Y}$ direction. The gradient magnitude is given by:

$$
\mathbf{G}=\sqrt{\mathbf{a}_{10}^{2}+\mathbf{a}_{01}^{2}}
$$

Formula (22), represents the 2D design digital filter used to obtain image edges, we named this digital filter 'Raafat filter'.

\section{REVIEW OF EDGE DETECTOR OPERATORS}

\section{Sobel Operator}

The Sobel operator is based on convolving the image with a small, separable and integer valuated filter in the horizontal and vertical directions, and therefore is relatively inexpensive in terms of computations. On the other hand, the gradient approximation that it produces is relatively crude, in particular for high frequency variations in the image. A 2D spatial gradient measurement can be imple- 
mented by using Sobel operator on an image (Sobel, 1990). The approximate absolute gradient magnitude at each point (pixel) can be computed using this edge detector. The $3 \times 3$ Sobel operator which is widely used for edge detection of different images is given as:

$$
\begin{aligned}
& \begin{array}{lll}
-1 & 0 & +1
\end{array}
\end{aligned}
$$

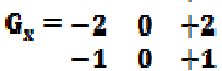

$$
\begin{aligned}
& +1+2+1 \\
& \begin{array}{lll}
-1 & 0 & +1
\end{array} \\
& \mathbf{G}_{y}=\begin{array}{ccc}
+1 & +2 & +1 \\
-1 & -2 & -1
\end{array}
\end{aligned}
$$

where $G_{x}$ and $G_{y}$ are the gradient in the $X$ direction and $\mathrm{Y}$ direction respectively. The gradient magnitude is given by:

$$
\mathbf{G}=\sqrt{\mathbf{G}_{x}^{2}+\mathbf{G}_{y}^{2}}
$$

\section{Roberts Operator}

Roberts operator is to approximate the gradient of an image through discrete differentiation which is achieved by computing the sum of the square of the differences between diagonally adjacent pixels. The result will highlight changes in intensity in diagonal direction. The Roberts Cross operator performs a simple, 2D spatial gradient measurement on an image (Roberts, 1965). Pixel values at each point in the output represent the estimated absolute magnitude of the spatial gradient of the input image at that point. The Roberts operator consists of a pair of $2 \times 2$ convolution kernels given as:

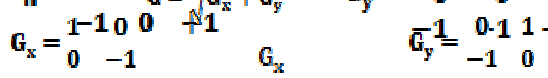

where $G_{\mathrm{g}}$ and $\mathrm{G}_{\mathrm{y}}$ are the gradient in the $\mathrm{X}$ direction and $\mathrm{Y}$ direction respectively. This is very similar to thesobel operator. The gradient magnitude is given by:

$$
\mathbf{G}=\sqrt{\mathbf{G}_{\mathrm{s}}^{2}+\mathbf{G}_{y}^{2}}
$$

\section{Prewitt Operator}

Prewitt operator is based on convolving the image with a small, separable and integer valuated filter in the horizontal and vertical directions. The gradient approximation with it produces in relatively crude. Prewitt op- erator is similar to the Sobel operator and is used for detecting vertical and horizontal edges in image (Prewitt, 1970). The 3x3 Prewitt operator is given as:

$$
\begin{aligned}
& \begin{array}{lll}
-1 & 0 & +1
\end{array} \\
& \mathrm{G}_{\mathrm{x}}=\begin{array}{lll}
-1 & 0 & +1 \\
-1 & 0 & +1
\end{array} \\
& \mathrm{G}_{y}=+1+1+1 \\
& \text { where } G_{\mathrm{x}} \text { and } G_{y} \text { are the gradient in the } X
\end{aligned}
$$
direction and $\mathrm{Y}$ direction respectively. The gradient magnitude is given by:

$$
\mathbf{G}=\sqrt{\mathbf{G}_{x}^{2}+\mathbf{G}_{y}^{2}}
$$

\section{Canny Operator}

Canny operator not give a good approximation of rotational symmetry and therefore gives bias towards horizontal and vertical edges. This operator is based on three criteria. The basic idea uses a Gaussian function to smooth image firstly (Canny, 1986). The maximum value of first derivative also corresponds to the minimum of the first derivative. In other words, both points with dramatic change of grayscale (strong edge) and points with slight change of grayscale (weak edges) correspond to the second derivative zero-crossing point. Thus these two thresholds are used to detect strong edges and weak edges. The fact that Canny algorithm is not susceptible to noise interference enables its ability to detect true weak edges. Canny defined optimal edge finding as a set of criteria that maximize the probability of detecting true edges while minimizing the probability of false edges. To smooth the image, the canny edge detector uses Gaussian convolution, is the spread of the Gaussian and controls the degree of smoothing. The $3 \times 3$ Canny operator is given as:

$$
\begin{aligned}
& \mathbf{G}_{x}=\begin{array}{rrr}
-1 & 0 & +1 \\
-2 & 0 & +2 \\
-1 & 0 & +1
\end{array} \quad \mathbf{G}_{y}=\begin{array}{rrr}
+1 & +2 & +1 \\
0 & 0 & 0 \\
-1 & -2 & -1
\end{array} \\
& \text { where } \mathbf{G}_{\mathrm{z}} \text { and } \mathbf{G}_{\mathrm{y}} \text { are the gradient in the } \mathrm{X}
\end{aligned}
$$
direction and $\mathrm{Y}$ direction respectively. The gradient magnitude is given by:

$$
\mathbf{G}=\sqrt{\mathbf{G}_{\mathrm{R}}^{2}+\mathbf{G}_{\mathrm{Y}}^{2}}
$$




\section{Laplacian of Gaussian (LOG) Operator}

The Laplacian is a 2D isotropic measure of the spatial derivative of an image (Saket, 2012). The Laplacian of an image highlights regions of rapid intensity change and is therefore often used for edge detection. The Laplacian is often applied to an image that has first been smoothed with something approximating a Gaussian smoothing filter in order to reduce its sensitivity to noise. The operator normally takes a single gray level image as input and produces another gray level image as output. The $3 \times 3$ Laplacian operator is given as:

$$
G_{x}=\begin{array}{rrr}
+1 & 0 & +1 \\
+1 & -8 & +2 \\
+1 & 0 & +1
\end{array} \quad G_{y}=\begin{array}{rrrr}
-1 & +2 & -1 \\
-1 & +2 & -1
\end{array}
$$

where $\mathbf{G}_{\mathrm{x}}$ and $\mathrm{G}_{\mathrm{y}}$ are the gradient in the $\mathrm{X}$ direction and $\mathrm{Y}$ direction respectively. The gradient magnitude is given by:

$$
\mathbf{G}=\sqrt{\mathbf{G}_{x}^{2}+\mathbf{G}_{y}^{2}}
$$

Laplacian, this filter is mainly used for corner detection and object recognition.

\section{APPLICATION OF EDGE ENHANCEMENT FILTERS}

Gabal Gattar batholith located at the northern Eastern Desert of Egypt has been chosen as a pilot area for application of edge enhancement process on its digital image. The filters used for edge enhancement are Sobel, Roberts, Prewitt, Canny, Laplacian and the Raafat calculated filter. The results were used to compare between filters.

\section{Geology of Gabal Gattar}

Gabal Gattar area is located to the west of Hurghada town, at a distance of about 35 $\mathrm{km}$ between lat. $26^{\circ} 52^{\prime} \mathrm{N}$ and $27^{\circ} 08^{\prime} \mathrm{N}$, and Long. $33^{\circ} 13^{\prime} \mathrm{E}$ and $33^{\circ} 26^{\prime} \mathrm{E}$ (Fig. 1). Gabal Gattar batholith has an oval shape of nearly $\mathrm{N}-\mathrm{S}$ trend. It shows geological curved and linear contacts with its surrounding rocks. These rocks from older are metasidements, diorrite, older granite, hammamat sediments, younger granites and wadi deposits (Fig. 2). The exposed rocks are mainly of felsic and mafic rocks which greatly different in their mineralogical composition accordingly show variety of gray levels. Figure (2) shows that the exposed rocks in the study area show various degrees of gray tones scale ranging from nearly white for wadi deposits to dark gray level for hammamat sediments, because they have different mineralogical composition.

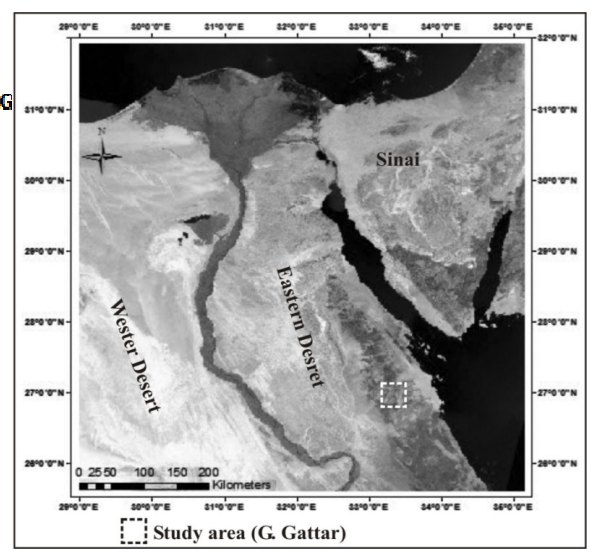

Fig. 1: Location map of study area (Gabal Gattar)

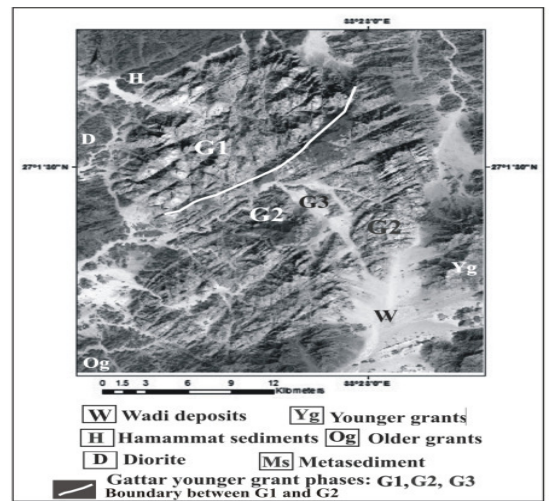

Fig. 2: Landsat Image of Gabal Gattar area, shows the granitic phases of Gattar batholith and its the surrounded rocks 
Rakaiby and Shalaby (1992) classified the Gattar granite into three granitic phases slightly different in their mineralogical composition, they are from younger G1, G2 and G3. They also different in their surface textures, relief, trends and density of faults, fractures and dykes. There are contacts between the three phases of granite of the batholiths. Field work and satellite image (Fig. 2) observations indicate that, the area has been subjected to multiphase deformation, due to a complex sequence of successive tectonic events. These phases are reflected on the G. Gattar batholith through a high density of faults and joints in different trends, depending on the causative stress tensors and the strength properties of granite material.

Lineaments analysis is carried out on 502 fracture lineaments interpreted from the landsat image (Fig. 2). The analysis of the lineaments numbers is summarized in Table (1) and represented on the rose diagram (Table 1) Geom. distr. The lineaments can be arranged according to their numbers in decreasing order as follows, Haridy, M. M. (2002):

NE-SW, ENE-WSW, N-S, NNW-SSE, NW-SE, NNE-SSW, E-W and WNW-ESE.

\section{Results After Applying Edge Operators On The Satellite Image Of G. GATTAR}

Figure (3) shows the gray scale image of the study area, where the Raafat filter and the

Table 1 : Analysis of photo-lineaments trends in Gabal Gattar batholith

\begin{tabular}{|c|c|c|c|c|}
\hline Order & Trends & Number & Number \% & \\
\hline 1 & NE-SW & 214 & 42.6 & \\
\hline 2 & ENE-WSW & 113 & 22.5 & \\
\hline 3 & $\mathrm{~N}-\mathrm{S}$ & 52 & 10.3 & \\
\hline 4 & NNW-SSE & 34 & 6.8 & \\
\hline 5 & NW-SE & 32 & 6.4 & \\
\hline 6 & NNE-SSW & 29 & 5.8 & Geometrical distribution \\
\hline 7 & E-W & 19 & 3.8 & of lineaments \\
\hline 8 & WNW-ESE & 9 & 1.8 & \\
\hline Total & & 502 & $100 \%$ & \\
\hline
\end{tabular}

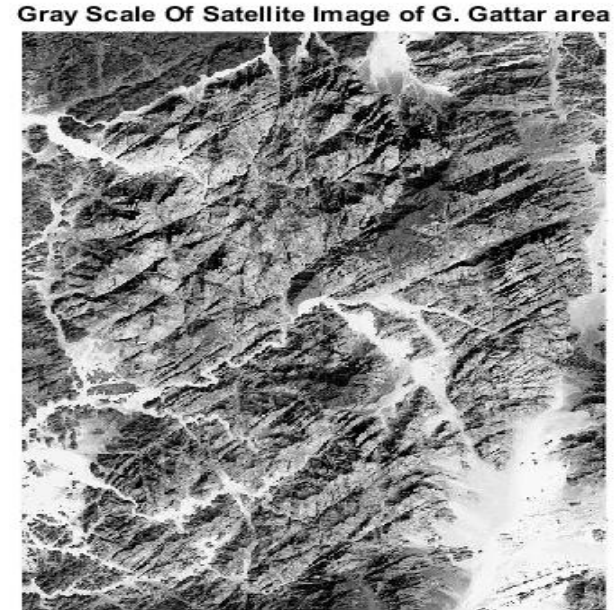

Fig. 3 : Gray Scale of Satellite Image of Gabal Gattar area

other filters were applied, while Figure (4), represents the results of applying the different edge operators on the landsat image of G. Gattar pluton batholith.

Comparing between the resulted images shows that, the edges enhancement using Raafat filter on the satellite image of G. Gattar is more evident and clearly obvious than the enhancement of the other filters. Raafat edge detector defined well the rock edges and boundaries between different rock types and become the efficient one among all other detectors used in comparison.

\section{CONCLUSIONS}

Figure (4) shows the results of application of edge enhancement techniques of the landsat image of G. Gattar, using Sobel, Roberts, Prewitt, Canny, Laplacian (Log) filters, as well as the Raafat filter, the following are the major characteristics of the enhanced images:

1-Sobel, Prewitt and Canny operators enhanced the vertical and horizontal lineaments.

2-Roberts operator enhanced the diagonal 


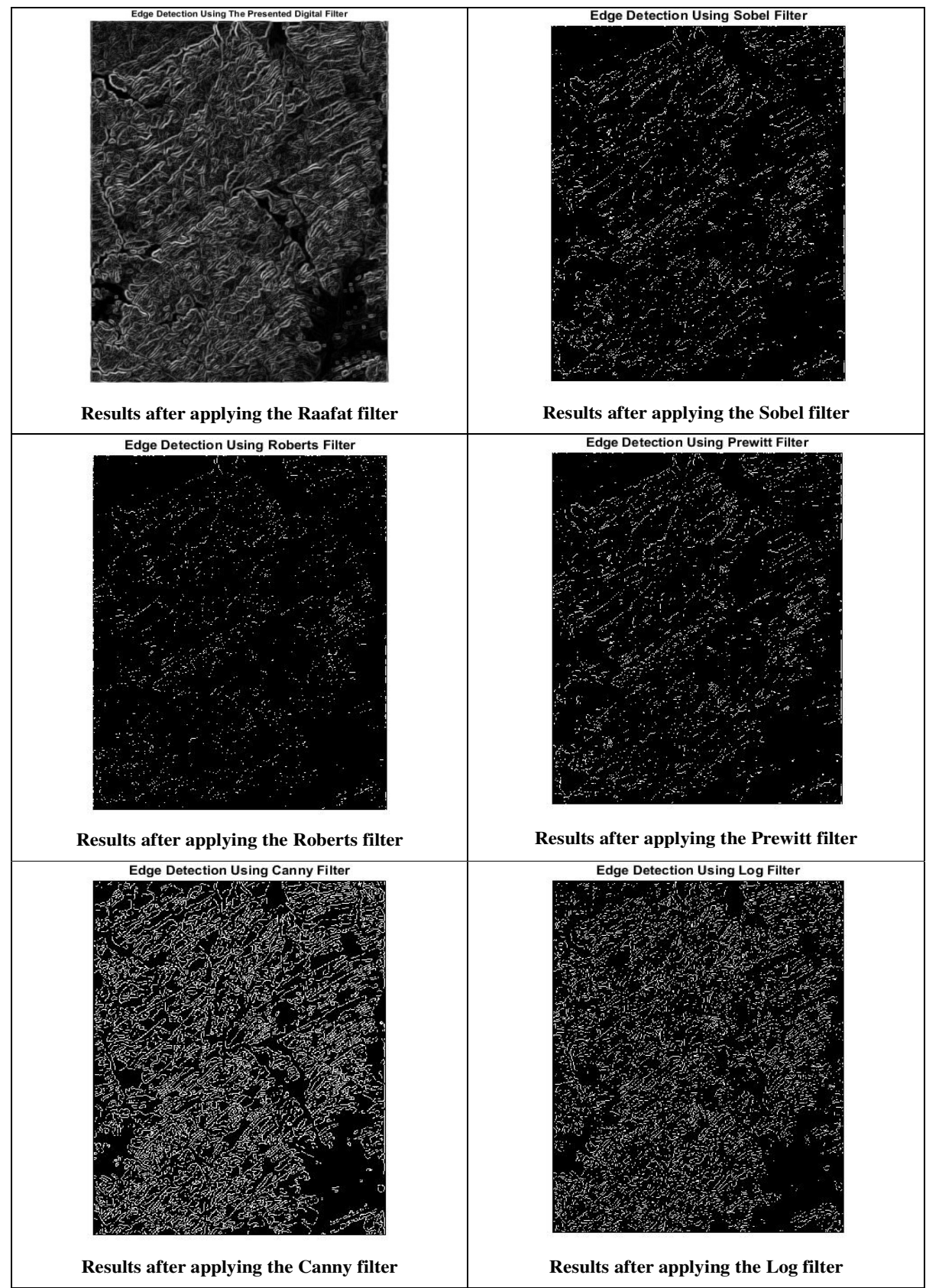

Fig. 4 : Edge Enhancement Images of Gabal Gattar 
lineaments, while Log operator enhanced the corner between lineaments.

3-The Raafat operator enhanced the curved lineaments accordingly pottery the Gattar batholith very well than the other filters.

4-The edge enhanced features are dissected and very curded in images of Canny and Log filters, according become difficult to interpret lineaments of different directions.

5-The edge enhanced features in Sobel and Prewitt, shows the ENE trend more obvious than other trends.

6-The edge enhanced features in Roberts are poorly identified.

7-The Raafat filter used $5 \times 5$ window, while the other filters used $3 \times 3$ window except Roberts filter used $2 \times 2$ window.

8-Geological contact between Gattar granite phases (G1 and G2) is easy to identified in the Raafat operator, while this contact is unclear in images of the other filters.

9-The enhanced lineaments in image of the Raafat filter are very clear, continuous, and sharply clear than these features enhanced by other filters.

\section{REFERENCES}

Canny, J., 1986. A Computational Approach to Edge Detection, IEEE Transactions on Pattern Analysis anad Machine Intelligence. 8, 679700 .
Haridy M.M., 2002. Geotechnical and geotectonic studies of G. Gattar U-deposits environs, Egypt, and its implications in localizing target structures for U-exploration. Ph.D. Thesis, Geo. Dept, Fac. Sci., Cairo Univ., Egypt.

Lillesand, T.M. and Kiefer, R.W., 2000. Remote sensing and image interpretation, Johnwiley sons, New York, 723p.

Prewitt, J., 1970. Object Enhancement and Extraction, Picture Processing and Psycho-pictorics (B. Lipkin and A. Rosenfeld, Ed.), NY, Academic Pres.

Rakaiby, M.L. and Shalaby M.H., 1992. Geology of Gebel Gattar batholith, Central Eastern Desert, Egypt. Inter. J. remote sensing, 13, No. 12, 2337-2347.

Roberts, L.G., 1965. Machine Perception of ThreeDimensional Solids, in optical and ElectroOptical Information Processing (J. Tippett, Ed.), MIT Pres, 159-197.

Sabins, F.F., 1996. Remote sensing, principles and interpretation. Remote sensing Enterprises. nc, New York.

Saket Bhardwaj, Ajay Mittal, 2012. A Survey on Various Edge Detector Techniques, Procedia Technology, 220-226.

Sobel, I., 1990. An Isotropic $3 \times 3$ Gradient Operator, Machine Vision for Three-Dimensional Scenes, Freeman, H., Academic Pres, NY, 376-379.

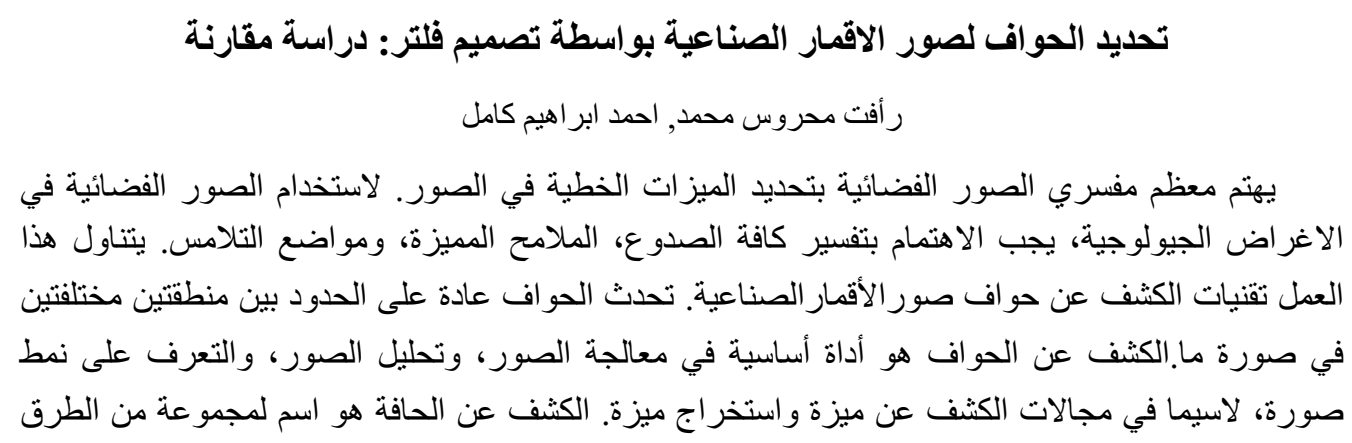


الرياضية التي تهدف إلى تحديد نقاط في صورة الأقمار الصناعية التي يتغير سطوع الصورة بشكل حاد، أو تحديدا

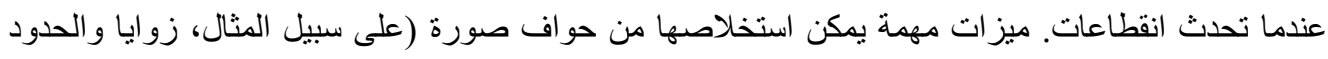
الجيولوجية، خطوط، منحنيات). هذا العمل يقدم تقنية كثف جديدة عن طريق تصميم فلتررقمي ثنائي الأبعاد و التي

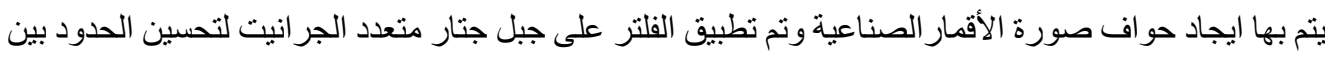

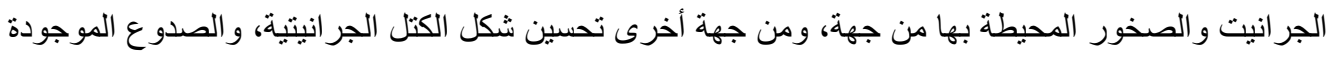

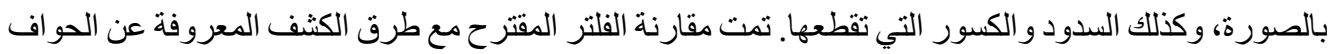

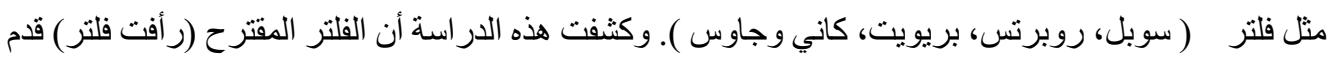
حواف أكثر وضوحا من الفلاتر الآخري. 